\title{
NOUVELLE
}

\section{IDO, une enzyme dangereuse pour l'obésité}

Ludivine Laurans, Soraya Taleb
Inserm U970, centre de recherche cardiovasculaire de Paris (PARCC), université Paris-Descartes, Hôpital Européen Georges Pompidou, 20, rue Leblanc, 75015 Paris, France. soraya.taleb@inserm.fr
> L'obésité, en constante augmentation, est désormais la plus importante pathologie dans les sociétés occidentales ayant pour origine la nutrition. Cette évolution est d'autant plus préoccupante que l'obésité constitue un facteur de risque important de diverses pathologies, comme le diabète de type 2 et les maladies cardiovasculaires. II est maintenant clairement établi que le tissu adipeux blanc n'est pas simplement un organe passif de stockage et de mobilisation des triglycérides. II est également un organe endocrine capable de produire de nombreuses molécules, appelées «adipokines», qui sont impliquées dans l'homéostasie énergétique et la physiopathologie de l'obésité et de ses complications. D'autres conséquences de l'obésité, plus récemment décrites, recouvrent la dysbiose intestinale et l'augmentation de perméabilité intestinale vis-à-vis de composants bactériens qui seraient impliqués dans les complications liées à l'obésité, comme I'insulino-résistance $[1,2]$. Toutes ces altérations aboutissent à une inflammation chronique, un déterminant commun à l'obésité et aux maladies cardiovasculaires. Cependant, les mécanismes exacts permettant d'établir un lien et d'expliquer la dérégulation des voies inflammatoires en cause dans le développement de ces maladies ne sont pas complètement identifiés.

L'indoléamine 2-3 dioxygénase 1 (IDO) est une enzyme dont l'expression est induite au cours de la réaction inflammatoire. Elle est responsable du catabolisme d'un acide aminé essentiel, le tryptophane (Trp), en $\mathrm{N}$-formylkynurénine, conduisant à la production de métabolites dérivés de la kynu- rénine (Kyn). Jusqu'à présent, il était admis que I'IDO exerçait des activités ayant des effets immunosuppresseurs, notamment via l'induction de lymphocytes $T$ aux propriétés régulatrices, les lymphocytes Treg $[3,4]$. Cependant, nos résultats publiés dans la revue Cell metabolism [5] ont montré que, contrairement à ce qui était attendu (étant donnée l'activité immunosuppressive de cette enzyme), l'absence d'IDO se traduit en fait par un effet athéroprotecteur, révélant le rôle plus complexe de cette enzyme.

\section{Rôle d'IDO dans l'obésité}

L'étude que nous avons menée sur cette enzyme dans le syndrome métabolique a été publiée récemment dans Nature Medicine [6]. Dans cette étude, nous avons montré que l'activité enzymatique d'IDO, estimée par le rapport Kyn/Trp, était augmentée dans le plasma, mais également dans les tissus adipeux blancs, le foie et le muscle de souris rendues obèses par un régime riche en lipides. Cette augmentation de l'activité d'IDO au cours de l'obésité suggère que cette enzyme aurait un rôle important dans le développement de la pathologie. L'analyse de souris génétiquement modifiées et n'exprimant plus le gène Ido a permis d'étayer cette hypothèse. En effet, les souris n'exprimant plus Ido sont protégées contre l'obésité et ses complications, telles que l'insulinorésistance. Nous avons obtenu des résultats similaires en utilisant un inhibiteur pharmacologique d'IDO, le méthyl-tryptophane (IMT), suggérant un rôle délétère de l'activité enzymatique d'IDO dans ce contexte. Afin de déterminer quels types cellulaires exprimant IDO étaient impliqués dans le phénotype de protection observé, des expériences ont été réalisées avec des souris chimères (des souris irradiées de façon sub-léthale et transplantées avec la moelle osseuse d'intérêt). Nous avons pu ainsi montrer que l'absence d'expression d'IDO spécifiquement dans le compartiment non myéloïde reproduisait le phénotype observé chez les souris n'exprimant plus IDO $\left(/ \mathrm{do}^{-/-}\right)$. Cette observation nous a conduit à émettre l'hypothèse que IDO, exprimée au niveau intestinal, pouvait être impliquée. En effet, au cours de l'obésité, une augmentation de l'activité IDO est observée dans l'intestin grêle et au niveau du côlon, suggérant son implication au niveau intestinal.

\section{Rôle d'IDO intestinal dans l'obésité}

Plusieurs observations réalisées chez des souris n'exprimant plus le gène Ido nous ont conduits à rechercher un rôle direct d'IDO sur le microbiote. Chez ces souris, nous avons en effet observé une modification de la flore intestinale avec, notamment, une diminution du rapport firmicutes/bactéroides qui, généralement, augmente au cours de l'obésité [7]. Ces modifications sont associées à une diminution de l'expression de gènes codant des molécules à activités inflammatoires au niveau intestinal, ainsi qu'à une élévation des taux d'acides gras à chaîne courte, connus pour leurs multiples effets bénéfiques [2]. Corroborant ces observations, les taux plasmatiques de lipopolysaccharides bactériens (LPS) chez les souris sont également abaissés, suggérant une meilleure perméabilité intestinale chez les souris dépourvues d'IDO au cours de l'obésité.

Afin d'examiner l'importance du microbiote dans ces réponses, nous avons traité les 


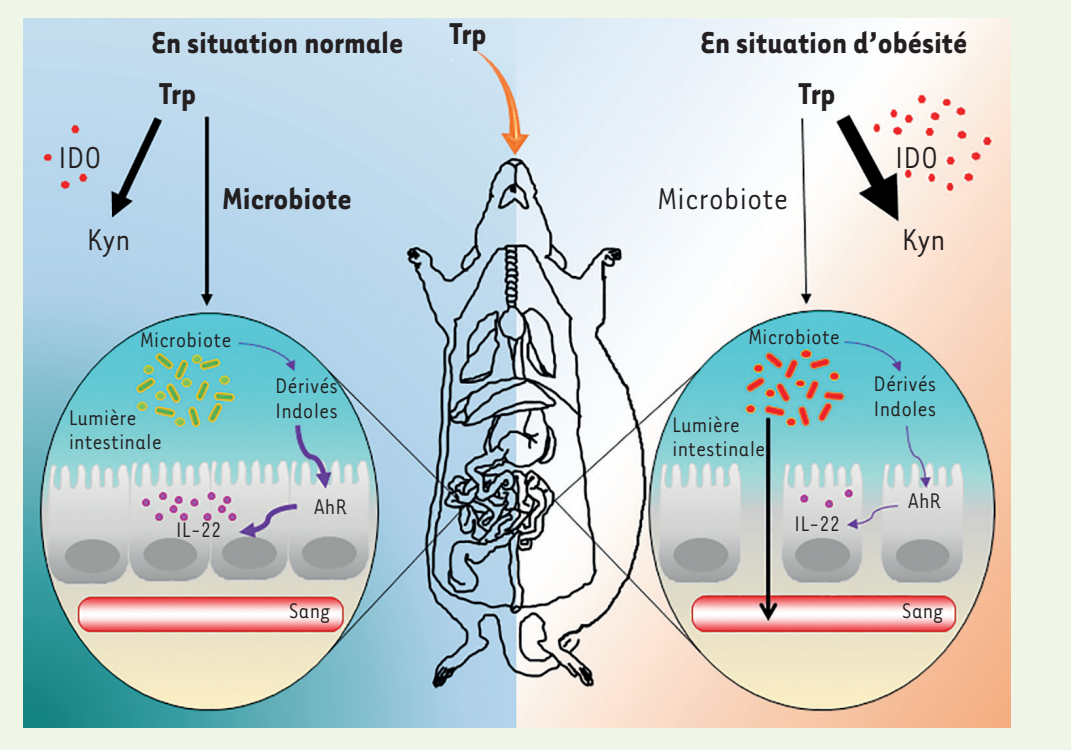

Figure 1. Métabolisme du tryptophane. En situation physiologique, le tryptophane (Trp) apporté par l'alimentation est converti en kynurénine (Kyn) par l'enzyme IDO (indoléamine 2-3 dioxygénase) et en dérivés indoles par un sous-type de bactéries conduisant à l'activation de l'AhR (aryl hydrocarbon receptor) et à une production d'interleukine-22 (IL-22), en particulier. En cas d'obésité, le métabolisme du Trp subit une déviation majeure vers la production de kynurénine (Kyn) au détriment de la voie des indoles, conduisant à une dysbiose et à une augmentation de la perméabilité intestinale.

souris dépourvues d'ldo par un cocktail d'antibiotiques permettant l'élimination de la flore intestinale. Ce traitement par antibiotiques annule la différence de poids observée entre les souris, suggérant l'importance du microbiote. Étayant ce résultat, le transfert de flore intestinale de souris obèses traitées avec l'inhibiteur d'IDO (le 1MT) protège les souris receveuses contre la prise de poids, démontrant l'importance du microbiote modifié par IDO dans la prise de poids. La recherche des mécanismes impliqués a montré l'importance de l'activité enzymatique d'IDO dans la modulation de la voie indole (Figure 1). Contrairement aux hypothèses formulées initialement, nos expériences ont montré qu'IDO ne jouait pas un rôle direct sur l'obésité via la génération de kynurénine, mais intervenait indirectement par la diminution des dérivés indoles. En situations physiologiques, le Trp est métabolisé par ID0, pour produire la Kyn, mais également par le microbiote, pour conduire à la production de dérivés indoles, tels que I'IAA (indole-3-acetic acid) [8]. Ces dérivés indoles sont des ligands de l'AhR (aryl hydrocarbon receptor), qui sont connus pour induire la production d'IL-22

(interleukine-22)

$(\rightarrow)$ Voir la Nouvelle $[9,10](\rightarrow)$ une cytokine importante de B. Lamas et al., $\mathrm{m} / \mathrm{s}$ $n^{\circ} 11$, novembre 2016, page 933

pour l'homéostasie intestinale qui protègerait contre l'obésité et ses complications [11]. Dans le cas d'obésité, l'augmentation de l'activité IDO induit cependant une modification du métabolisme du Trp en favorisant une production accrue de Kyn au détriment de celle des dérivés indoles et, in fine, de l'IL-22 (Figure I). Une activité IDO accrue est donc responsable d'une altération de l'écosystème intestinal, ce qui aura un impact négatif sur le syndrome métabolique. Confirmant ces résultats, nos données chez l'homme ont montré une augmentation de Kyn et une diminution d'IAA dans les fèces des sujets souffrant d'obésité et de diabète de type 2 .

\section{Conclusions et perspectives}

Nos résultats montrent donc, pour la première fois, un rôle direct d'IDO dans le syndrome métabolique, l'une des causes des maladies cardiovasculaires en augmentation. L'obésité est ainsi associée à une augmentation de l'activité enzymatique d'IDO avec, pour conséquence, une utilisation accrue du Trp pour générer la kynurénine au détriment des dérivés indoles et de l'IL-22. Cette étude permet donc d'envisager I'utilisation thérapeutique d'inhibiteurs d'IDO afin de lutter contre l'obésité. Un inhibiteur spécifique de cette enzyme a été testé jusqu'en phase 3. II n'a pas montré de bénéfice dans les traitements du cancer. Mais cette molécule pourrait être proposée pour le traitement des maladies cardio-métaboliques. $\diamond$ IDO, an enzyme that induces dangerously obesity

\section{LIENS D'INTÉRÊT}

Les auteurs déclarent n'avoir aucun lien d'intérêt concernant les données publiées dans cet article.

\section{RÉFÉRENCES}

1. Tang WHW, Kitai T, Hazen SL. Gut microbiota in cardiovascular health and disease. Circ Res 2017 ; $120: 1183-96$.

2. Schroeder BO, Bäckhed F. Signals from the gut microbiota to distant organs in physiology and disease. Nat Med 2016 ; 22 : 1079-89.

3. Mellor AL, Munn DH. IDO expression by dendritic cells: tolerance and tryptophan catabolism. Nat Rev Immunol $2004 ; 4: 762-74$.

4. Puccetti P, Grohmann U. IDO and regulatory T cells: a role for reverse signalling and non-canonical NF-kappaB activation. Nat Rev Immunol 2007 ; 7 : 817-23.

5. Metghalchi S, Ponnuswamy P, Simon T, et al. Indoleamine 2,3-dioxygenase fine-tunes immune homeostasis in atherosclerosis and colitis through repression of interleukin- 10 production. Cell Metab $2015 ; 22: 460-71$.

6. Laurans L, Venteclef N, Haddad Y, et al. Genetic deficiency of indoleamine 2,3-dioxygenase promotes gut microbiota-mediated metabolic health. Nat Med 2018 ; 24 : 1113-20. doi : 10.1038/s41591-018-0060-4

7. Turnbaugh PJ, Ley RE, Mahowald MA, et al. An obesityassociated gut microbiome with increased capacity for energy harvest. Nature 2006 ; 444 : 1027-31.

8. Lamas B, Richard ML, Leducq V, et al. CARD9 impacts colitis by altering gut microbiota metabolism of tryptophan into aryl hydrocarbon receptor ligands. Nat Med 2016; $22: 598-605$.

9. Zelante T, lannitti RG, Cunha C, et al. Tryptophan catabolites from microbiota engage aryl hydrocarbon receptor and balance mucosal reactivity via interleukin-22. Immunity 2013; 39: 372-85

10. Lamas B, Richard ML, Sokob H. Card9 et colyte : un pont entre dysbiose et immunité. Med Sci (Paris) 2016 ; 32 : 933-6.

11. Wang $X, 0$ ta N, Manzanillo P, et al. Interleukin-22 alleviates metabolic disorders and restores mucosal immunity in diabetes. Nature $2014 ; 514$ : 237-41. 